\title{
Some remaining important questions after LUNG SAFE
}

\author{
Discussion of "Potentially modifiable factors contributing to outcome from acute \\ respiratory distress syndrome: the LUNG SAFE study"
}

Didier Dreyfuss ${ }^{1,2,3}$, Stéphane Gaudry ${ }^{1,2,4}$, Fabiana Madotto ${ }^{5}$ and John G. Laffey $6,7,8, *^{*}$

๑ 2017 Springer-Verlag Berlin Heidelberg and ESICM

\section{Comments from Drs Dreyfuss and Gaudry}

The LUNG SAFE study is a major advance in the understanding of epidemiology and prognosis factors of ARDS [1].

The "Discussion" states that the use of higher PEEP in moderate or severe ARDS was independently associated with improved hospital survival, suggesting "benefits with higher PEEP particularly in more severe ARDS". This assertion is disputable:

- Figure 2 shows that higher PEEP was associated with improved survival only in moderate ARDS. Moreover, this observation is at variance with the results of a meta-analysis which found beneficial effects of higher PEEP in both moderate and severe ARDS [2].

- There was no benefit of higher PEEP on ICU mortality (see "supplementary appendix"). It is puzzling why a baseline PEEP level affected hospital mortality but not ICU mortality.

- The authors equate statistical and causal links. The association of higher PEEP with survival might reflect the fact that patients with better prognosis tolerated higher PEEP.

- The authors "forced" PEEP in their multivariate analysis. Though acceptable this is not specified in the "Methods", which indicate that only variables with $P<0.05$ on bivariate analysis were entered in multivariate analyses. $P$ values for association of PEEP with mortality were $>0.6$.

\footnotetext{
*Correspondence: laffeyj@smh.ca

${ }^{6}$ Department of Anesthesia and Critical Care Medicine, Keenan Research Centre for Biomedical Science, St Michael's Hospital, Toronto, Canada
} Full author information is available at the end of the article
Analysis of patients with true plateau pressure measurement deserves more attention to the respective interest of driving and plateau pressures. Both parameters predicted mortality with acceptable accuracy and neither seemed superior. LUNG SAFE suggests that pending an adequate prospective study, the two parameters should be closely monitored. The advantage of plateau pressure is that it requires no computation. Clinicians should not consider that dynamic distension (reflected by driving pressure) is the major culprit in ventilator-associated complications. Maximal distension, irrespective of driving pressure, is an important contributor to both the generation of ventilator-induced lung injury and mortality [3]. Physicians should simply monitor plateau pressure and adequately use early muscle paralysis and prone positioning in severe cases. LUNG SAFE brilliantly shows that these simple tools are too rarely used.

\section{Reply from Drs Madotto and Laffey}

We used hospital mortality because it is a robust and patient-centred parameter. This was a predefined choice, reported as the major outcome across the LUNG SAFE studies $[4,5]$.

We reported multivariable analyses relating to PEEP and hospital mortality. Higher PEEP [OR 0.95 per $\mathrm{cmH}_{2} \mathrm{O}$ PEEP (95\% CI 0.92-0.98); $P=0.001$; Table 2] was independently associated with better outcome in all patients with ARDS. When we dichotomized the dataset into lower versus higher PEEP, only patients with moderate ARDS that received lower PEEP $\left(<12 \mathrm{cmH}_{2} \mathrm{O}\right)$ had a higher risk of hospital mortality. In contrast to the authors' contention, we found that higher PEEP was associated with improved ICU survival (Table E2). 
We agree that an observational study permits no causal inferences regarding the relationship between PEEP and outcome. The association between PEEP and hospital mortality was independent of other factors included in the multivariate analysis, including multiple illness severity indices.

We did not 'force' PEEP into the multivariable analysis, but selected variables on the basis of stepwise regression, evaluating at each step the statistical significance of all "candidate" predictor variables, until there was no justification for entering or removing more (see "Methods"). Therefore, PEEP statistical significance was always checked during the stepwise "process" even if this variable showed a $P$ value exceeding 0.05 initially.

We entirely agree on the need for more routine measurement of plateau pressure. In regard to the relative merits of plateau versus driving pressures, we prefer to leave this debate to others. In LUNG SAFE, when both measurements were included in multivariable analysis, plateau pressure was no longer associated with outcome, likely due to colinearity (Table E4). When driving pressure was excluded, plateau pressure was associated with outcome (Table E5). It is indeed important to consider the potential for both static and dynamic distension to cause harm, although we did not address this specific issue.

\footnotetext{
Author details

${ }^{1}$ AP-HP, Service de Réanimation Médico-Chirurgicale, Hôpital Louis Mourier, 92700 Colombes, France. ${ }^{2}$ INSERM IAME UMR 1137, 75018 Paris, France. ${ }^{3}$ Univ Paris Diderot, IAME, UMR 1137, Sorbonne Paris Cité, 75018 Paris, France. ${ }^{4}$ Univ Paris Diderot, ECEVE, UMR 1123, Sorbonne Paris Cité, 75018 Paris, France.

${ }^{5}$ Research Center on Public Health, School of Medicine and Surgery, University of Milano-Bicocca, Monza, Italy. ${ }^{6}$ Department of Anesthesia and Critical Care Medicine, Keenan Research Centre for Biomedical Science, St Michael's Hospital, Toronto, Canada. ${ }^{7}$ Department of Anesthesia, University of Toronto, Toronto, Canada. ${ }^{8}$ Department of Physiology, University of Toronto, Toronto, Canada. ${ }^{9}$ Interdepartmental Division of Critical Care Medicine, University of Toronto, Toronto, Canada.
}

\section{Compliance with ethical standards}

Conflicts of interest

The authors declare no conflict of interest. This article does not contain any studies with human participants or animals performed by any of the authors.

Accepted: 28 January 2017

Published online: 17 February 2017

\section{References}

1. Laffey JG, Bellani G, Pham T et al (2016) Potentially modifiable factors contributing to outcome from acute respiratory distress syndrome: the LUNG SAFE study. Intensive Care Med 42:1865-1876. doi:10.1007/ s00134-016-4571-5

2. Briel M, Meade M, Mercat A et al (2010) Higher vs lower positive end-expiratory pressure in patients with acute lung injury and acute respiratory distress syndrome: systematic review and meta-analysis. JAMA 303:865-873

3. Dreyfuss D, Ricard J-D, Gaudry S (2015) Did studies on HFOV fail to improve ARDS survival because they did not decrease VILI? On the potential validity of a physiological concept enounced several decades ago. Intensive Care Med 41:2076-2086. doi:10.1007/s00134-015-4062-0

4. Bellani G, Laffey JG, Pham T, Fan F, Brochard L, Esteban A, Gattinoni L, van Haren F, Larsson A, McAuley DF, Ranieri M, Rubenfeld GD, Thompson BT, Wrigge H, Slutsky AS, Pesenti A, LUNG SAFE Investigarors and ESICM Clinical Trials Group (2016) Epidemiology, patterns of care, and mortality for patients with acute respiratory distress syndrome in intensive care units in 50 countries. JAMA 315:788-800

5. Bellani G, Laffey JG, Pham T, Madotto F, Fan E, Brochard L, Esteban A, Gattinoni L, Bumbasirevic V, Piquilloud L, van Haren F, Larsson A, McAuley DF, Bauer PR, Arabi YM, Ranieri M, Antonelli M, Rubenfeld GD, Thompson BT, Wrigge $H$, Slutsky AS, Pesenti A, on behalf of the LUNG SAFE Investigators, and the ESICM Trials Group (2016) Non-invasive ventilation of patients with ARDS: insights from the LUNG SAFE study. Am J Respir Crit Care Med 195:67-77 